\title{
UYGHURLARDIKI MUQEDDES SANLARGHA MUNASIWETLIK ADETLER HEQQIDE IZDINISH
}

\author{
[Araştırma Makalesi / Research Article]
}

\section{Qisqiche Mezmuni}

Uyghurlarning turmushida xasiyetlik sanlar chüshenchisi mewjut bolup, bu xasiyetlik sanlar bir qatar örp-adetlerning kélip chiqishi we dawamlishishigha sewep bolghan. Bu seweptin uyghur örp-adetlirini tetqiq qilghan waqtimizda xasiyetlik sanlargha sel qarashqa bolmaydu. Xasiyetlik sanlar chüshenchisi turmushning herqaysi sahelirige singip ketken. Xasiyetlik sanlargha baghlanghan étiqadi qarashlar we adetler Uyghurlarning nezir-chiraq pa'aliyetliride köprek körülidu. Nezir pa'aliyiti uyghurlarning ölüm murasimidiki intayin muhim qisim hésablinidu. Uyghurlar turmushida üch, yette, yigirme, qiriq we yil nezirisi ötküzidighan adet yéqinqi yillarghiche dawam qilghan idi. Bu maqalide Uyghurlar arisidiki üch, yette, yigirme we qiriq sanlirining muqeddes sanlar bolishidiki sewebler tehlil qilinidu. Maqalide yene xasiyetlik sanlarning kélip chiqishi, uyghurlar turmushigha singip kirishi we herxil murasim sheklide Uyghur medeniytining bir qismigha aylinish jeryani échip birilidu. Shundaqla bu xasiyetlik sanlargha baghlanghan örp-adetlerning shekillinish we dawamlishish tarixi yorutulup bérilidu. Maqalide yene xasiyetlik sanlar mewjut bolghan medeniyet amillirighimu köz yügürtüldi. Bu seweptin uyghurlar étiqad qilghan herxil dinlardiki xasiyetlik san chüshenchisi qisqiche halda analiz qilindi. Bu arqiliq Uyghurlarning örp-adetliri bilen Shamanizm chüshenchiliri arisidiki munasiwetni échip bérishke tirishilidi.

Achquchluq Sözler: Uyghurlar, Muqeddes Sanlar, Ölüm Murasimliri, Étiqad, Shamanizm.

\section{UYGURLARDAKİ KUTSAL SAYILARLA İLGILII ÖRF ADETLER ÜZERINNE BİR İNCELEME}

\author{
$\ddot{O} z$
}

Uygurlar, yașamın her alanında kutsal sayı kavramına sahiptir ve bu kutsal sayılar, bazı kültürel geleneklerin ortaya çıkmasına ve devam etmesine yol açmıștır. Bu yüzden Uygur örf adetlerini araştırırken kutsal sayıları ihmal etmemek gerekmektedir. Kutsal sayı düșüncesi Uygur kültürünün her alanında bulunmaktadır. Kutsal sayılarla ilgili inanç ve gelenekler, bir kişinin ölümünden sonraki üçüncü gün, yedinci gün, kırkıncı gün ve yıl dönümü gibi günlerde gerçekleştiren anma ve yas tutma faaliyetlerinde daha yaygındır. Uygur geleneklerine baktığımızda ölünün üç, yedi, yirmi, kırık ve yıl dönümlerinde yapılan yas tutma ve yemek ikramı merasimlerinin günümüze değin devam etmekte olduğunu öğrenmekteyiz. Bu makalede, Uygurlar arasındaki üç, yedi, yirmi ve kırk sayılarının neden kutsal sayılar olduğu analiz edilmiștir. Bu kutsal sayıların ortaya çıkışı ve merasim şeklini alarak Uygur kültürün bir parçası haline geldiği üzerinde durulmuştur. Aynı zamanda, kutsal sayıların tarihsel kökenlerini tespit etmek amaçlanmıştır. Makalede yine kutsal sayıların gelişiminde önemli rol oynayan kültürel unsurlara yer verilmiștir. Dolayısıyla Uygurlar terihten bugüne kadar inanmış olduğu dinlerde görülen kutsal sayı düşüncesi kısaca ortaya konulmuştur.

\footnotetext{
*Dr., e-posta: mutellipiqbal@gmail.com Orcid:0000-0003-3737-2345
} 
Şamanizm gibi dinî inançların Uygurların kültürel hayatında görülen kutsal sayılar kavramının oluşmasında büyük etkisi olduğu sonucuna varılmıştır.

Anahtar Kelimeler: Uygurlar, Kutsal Sayıları, Ölüm Törenleri, İnançlar, Şamanizm.

\title{
A STUDY OF THE RELATION BETWEEN CUSTOMS AND SACRED NUMBERS AMONG THE UYGHURS
}

\begin{abstract}
Uyghurs has the concept of sacred numbers in all walks of life, and these sacred numbers have led to the emergence and continuation of a few cultural traditions. Therefore, while researching Uyghur customs, it is necessary not to neglect the sacred numbers. The beliefs of the sacred number are ingrained in every aspect of cultural life. The customs regarding sacred numbers are more common in memory of the dead and mourning activities such as the dead's third, seventh, fortieth, and year's commemoration. When we observe the Uyghur tradition, we learn that the Nezir (a feast to provide for the poor) activities have continued until recently. This article analyzes the three, seven, twenty, and forty and their historical as well as religious sacredness. The emergence of these sacred numbers and the reasons for being part of the Uyghur culture as a ceremony were discussed. It also aims to explore the multicultural origins of sacred numbers. The article also includes cultural elements, which play an important role in the development of sacred numbers. Meanwhile, the idea of sacred numbers in various religions, which Uyghurs have believed in their history, has been put in discussion briefly. It is concluded that religious beliefs, such as Shamanism, played a great role in formation of the concept of sacred numbers in Uygur lives.
\end{abstract}

Keywords: Uyghurs, Sacred Numbers, Death Customs, Beliefs, Shamanism.

\section{Kirish}

Uyghurlar dunyadiki bashqa xelqlerge oxshashla özgiche medeniyet tarixigha ige qedimki milletlerning biri. Uyghurlarning medeniyet tarixini waraqlisaq, bezi medeniyet hadisilirining tarixining tulimu uzun ikenliki we bashqa xelqlerning bezi medeniyetliri bilen qismen oxshashliqini bayqaymiz. Jümlidin Uyghurlarda bashqa milletlerge oxshashla xasiyetlik san chüshenchisi mewjut bolghan we xasiyetlik sanlargha munasiwetlik bezi örpadetler hazirgha qeder dawamliship kelgen. Biz bu maqalide Uyghurlar turmushidiki xasiyetlik sanlar we bu xasiyetlik sanlarning Uyghur örp-adetliri bilen bolghan baghlinishi heqqide toxtilimiz.

Xasiyetlik sanlarni ulughlash aditi dunyaning herqaysi millet yaki xelqler arisida oxshash bolmighan derijide mewjut bulup, bu xil adetning peyda bolushi we tereqqi qilishi bir qeder murekkep arqa körünüshke ige. Uning üstige herqaysi xelqlerning diniy étiqadining oxshash bolmasliqi tüpeyli sanlarni ulughlash adetliridimu perqler mewjut. Mesilen Yaponlar yette sanini bextsizlik élip kilidighan san dep qaraydu. Biraq yette Türk xelqlirining étiqadida qedimdin buyan ulughlinip kelgen sanlarning biridur.

Bezi tetqiqatchi-alimlar sanlarni ulughlash qarishini iptida'i étiqad we qedimki sadda astronomiyelik chüshenchidin kelgen dep qaraydu. Uyghur turmushidiki nezir-chiraq adetlirining menbesi heqqide izden'gen tetqiqatchi Raxman Abduréhim mundaq yazidu: "Uyghurlar adette a'ilisi ichide ölüp ketkenlerni depne qilghan künidin hésablap üchinchi, yettinchi, yigirminchi, qiriqinchi, yüzinchi künliri we bir yili toshqan küni kala yaki qoy öltürüp uruq-tughqan, el-jama'etke ölüp ketken a'ile ejdadining rohigha atap nezir ötküzidu. Kishilerning chüshenchiside öltürülgen malning rohi merhum -ejdad rohigha 
mensup bolidu... Mana bularni Uyghurlarning Shaman dini étiqadidiki ejdad rohigha choqunushning qalduq tesiri dep qarashqa bolidu." (Abduréhim, 2006: 190).

Shunisi iniqki, Uyghurlarning nezir-chiraq, ölüm-yitim adetliride we turmush murasimlirida xasiyetlik sanlargha munasiwetlik nurghunlighan köz-qarash we adet xaraktérlik hadiseler mewjut. Biz bu maqalede üch, yette, yigirme, qiriq qatarliq bir qatar özgiche sanlarning kélip chiqishini tehlil qilish arqiliq Uyghurlar turmushidiki bezi adetlerning xaraktérini tehlil qilip chiqimiz. Bu arqiliq Uyghurlarning örp-adetliri bilen étiqadiy chüshenchiliri arisidiki munasiwetni échip bérishke tirishimiz.

\section{Xasiyetlik Sanlargha Baghlanghan Adetler}

Hemmige melum, Uyghurlar tarixta Islam dinini qubul qilishtin ilgiri Xiristiyan dinining tarmiqi bolghan Néstoriyan mezhipi, Budda dini, Zara'astir dini, Mani dini qatarliq bir qatar dinlargha ishen'gen we diniy medeniyetlerni yaratqan. Shunglashqa, Uyghurlardiki xasiyetlik sanlar chüshenchisi köp xil din we medeniyet amilliri bilen munasiwetlik bolmaqta.

Üch- Uyghurlarning qedimqi diniy étiqad qarishida muhim urun tutatti. Shunglashqa üchke munasiwetlik herxil adetler, maqal-temsiller, perhiz qarashlar Uyghurlarning turmushida hélihem mewjut. Uyghurlarning diniy étiqad tarixini waraqlaydighan bolsaq, üchke munasiwetlik bezi qarashlarni uchritishqa bolidu.

Islam dini eqidisige qarisaq, üchke munasiwetlik bezi uchurlarning barliqini bilimiz. Muxtar Yaqup bu heqte: “Qur'ani kerimde Allata'alaning 99 ismi bar bolup bu üchning 33 hessige teng bolidu. Musulmanlar taharet alghanda ezalarni üch qétimdin yuyidu. Musulmanlar rokuda üch qétimdin durut oquydu. Musulman kishining qérindishigha üch kündin artuq adawet tutmasliqi tekitlinidu. Teswining uruq sani 33 bolidu yaki 99 uruqi bar. Teswide üch bughum bulup, herbir bughum arisida 33 uruq bulidu. Musulmanlar seper yaki aghriq sewebidin roza tutalmay qalsa, roza héyt bayrimidin üch kün ötküzüp, andin kem qalghan rozini tutush belgilen'gen..."dep yazidu (Yaqup, 1999:80). Démek, yuqiriqi bayandin körünüp turuptuki, "üch" musulmanlarning ishlarni bir terep qilishida "chek-chégra yaki möhlet" meniside ishlitilgen.

Tetqiqatchi Raxman Abduréhim Uyghurlardiki üch étiqadchiliqi heqqide mundaq yazidu: "Üch sani dunyadiki herqaysi qedimiy milletlerde omumyüzlük ulughlinidighan 'xasiyetlik san'larning biri. Bu san yalghuz Uyghurlardiki qalduq shaman dini pa'aliyetliridila emes, belki ularning turmush adetliridimu muhim orunda turidu... Uyghurlarning Shaman dini chüshenchiside, pütkül alem-asman (yuruqluq dunyasi), yer (hayatliq dunyasi) we yer asti (qarangghuluq dunyasi) dep üchke bölün'gen." (Abduréhim, 2006: 266).

$\mathrm{Bu}$ yerde shuni qisturup ötüsh kérekki, Shaman dini Uyghurlarning iptida'iy étiqadida muhim orun tutidu. Uyghurlarning qedimqi ejdadliri Xiristiyan, Budda, Mani qatarliq dinlargha ishinishtin ilgiri Shaman dinigha ishen'gen. Shaman dini köp xudaliq din idi. Eng deslepki insanlarning özi we tebi'et dunyasi heqqidiki chüshenchisi chongqur bolmighachqa, tebi'et dunyasidiki nurghun nersilerni ulughlighan. Jümlidin sanlar sistémisimu ularning neziride bir xil séhirlik hadise hésablinatti. Shunglashqa sanlarni ulughlash aditi Shaman dinining diniy idiyesi bilen qoyuq munasiwetke ige idi. Shaman dinining muntizim paydilinidighan muqeddes kitabi bolmighachqa, üch étqadchiliqini Shaman dini bilen baghlap chüshinishte ilmiy asas tépish bir qeder qiyin. Emma Shaman dini tarixi intayin uzun din bolghachqa, Uyghurlarning turmushidiki nurghun medeniyet hadisilirining yiltizi bu din bilen baghlinishliq.

Uyghurlarning chöchek we maqal-temsilliride üchke munaswetlik nurghun misallar bar. Mesilen, xelq chöchekliride "üch hurun", "üch aka-uka”, "üch hünerwen”, "üch tépishmaq", "üch tal chach", "üch musapir"... digendek ibariler köp uchraydu. Maqal-temsillerde 
"mertem mertem üch mertem", "toshqan tirisimu üch kün chidaptu", "er oghli üch" digendek ibariler köp uchraydu.

Biz qedimki Turpan-Idiqut höjjetlirige qaraydighan bolsaq, üchni ulughlashqa munasiwetlik bezi mezmunlarni uchritimiz. Üch Uyghurlarning qedimqi iqtisadiy turmushigha xéli chong tesir körsetken. "Üch ordigha te'elluq yerler...eger xojidar yaxshi qarimighan bolsa üch yüz derre urup gunahi sürüshtürülidu. ...̈̈ch parche aq tawarni alte baqirgha alduq...üch ölüm uzatqan chaghdiki xirajet öydin yetmey ösümige..." (Turdi, 2002: 42). Bu bayanlardin körünüp turuptiki, üch nezirisi ötküzüsh Uyghurlarda islam dinini qubul qilishtin ilgirila omumliship bolghan. Üch ölüm uzatqan chaghdiki xirajetning yetmesliki heqqidiki bayan buninggha toluq delil bolalaydu. Uyghurlar arisida bezi jaylarda yéqinqi yillarghiche üch nezirisi ötküzidighan ehwal mewjut idi.

Uyghurlar üchni axirqi chek meniside qollinilidu. Birersi birer ishta meghlup bolsa, üchinchi qétimghiche sinaydu. Herqandaq ishning chékini üch ichide kontrol qilishni layiq köridu. Omumen qilip éytqanda üchning Uyghurlar turmushigha bolghan tesiri chongqur bolup, üch étqadchiliqining Uyghurlar bügünki kündimu ötküzüp kéliwatqan "üch nezir" aditi bilen roshen baghlinishliqi bar.

Yette sanimu üchke oxshashla dunyadiki xéli köp milletlerning xasiyetlik sanlirining biri. "Iptida'iy tepekkur" namliq kitabta Chilojlarning töt we yettini xasiyetlik san hésablaydighanliqi tonushturulghan. Bu kitabta yene Assiriye Babilunliqlarning yettini ulughlaydighanliqi, Malayaliqlarning périxonluq pa'aliyetliride yettining alahide menige ige ikenliki yézilghan (Bolwér, 1981:214). Biz Uyghur baxshilirining shamanliq pa'aliyetlirige qaraydighan bolsaq, périxonluq pa'aliyti jeryanida yettige munasiwetlik adet jeryanlirining köplükini bayqaymiz. Yette Uyghur baxshilirining pa'aliyitide muhim xasiyetlik sanlarning biri. Hindi dinida jesetni köydürüp bolghandin kéyin, jeset köydürülgen jaygha 49ni yézip qoyidighan adet bolghan. Bolwér bu xil adetning yettining hessilik sanidin kélip chiqqanliqini otturigha qoyidu (Bolwér, 1981: 214).

Yette Uyghurlardiki muqeddes san bolupla qalmastin, dunyaning bashqa jayliridiki xelqlerde qedimdin buyan muqeddes hésablinip kelgen. Irnisit Kasiri bu heqte mundaq yazidu: "yette reqimi téximu yüksek pellide oxshashla qedir-qimmetke sazawer, u Méssopotamiye kishilirining deslepki medeniyitidin etrapqa taralghan. Hetta, BabilonAssiriye kishilirining tesiri bolmighan yaki tesiri bolushi mumkin bolmighan rayonlardimu yette reqimi alahide muqeddes san" (Kasiri, 2001: 57). U yene mundaq yazidu: "Ottura esirlerde Xiristiyan dinidiki poplar yette reqimini tolghan takamul san dep qarap 'yette mukemmel sandur' déyishetti." (Kasiri, 2001: 57). Shunga shundaq éytishqa boliduki, yettining xasiyetlik san bulup chiqishi bir qeder murekkep arqa körünüshke ige.

Biz tarixiy yadikarliqlirimizni axtursaq, Uyghurlarning yettini xasiyetlik sanlar qatarida ulughlap, uninggha choqunghanliqini bayqaymiz. Qedimde yette ejdadlirimizning étiqad obiyktilirining muhim bir qisimi bolghan. Cherchendin tépilghan qedimiy qebriler we axiretlik buyumlarning köpinchisi Uyghurlarning yettini xasiyetlik sanlar qatarida ulughlighanlqini ispatlap béridu. Könchi deryasi boyidiki qedimiki qebriler xuddi quyash nur chéchiwatqandek halette orunlashturulghan bolup, qebre etrapigha nurghunlighan yaghach qozuqlar qéqilghan. Qozuqlar qebrini aylandurup qéqilghan bulup, chember shekilde sirtqa kéngeygen. Kishining diqqitini tartidighini shuki, qebrilerning etrapigha qéqilghan qozuqlarning chember sani yette bolghan. Qebridin chiqqan axiretlik buyumlarda tipik halda yettini muqeddes bilishning iznaliri saqlanghan. Mesilen, qebridin chiqqan yaghach heykelning yüzi yette qat yung yip bilen baghlanghan. Yaghach taghaqlarning köpinchisining chishi yette bolghan. Ilghuchlarning destisige yette dane shekil chiqirilghan. Qebirilerdin yene bilekke salidighan yette dane bileyzük chiqqan. (Liu Xuetang, 2009: 87). Cherchen qebirilirining yil dewri omumyüzlük halda buningdin 3800 
yil ilgiri dep jezimleshtürüldi. Démek, yettini xasiyetlik san dep ulughlash qarishining tarixi uzun bulup, Uyghurlarning iptida'iy étiqad éngi bilen munasiwetlik dep qarashqa bolidu.

Yettini ulughlash qarishi klassik eserlerdimu köp uchraydu. Menggü tash tékistliridin yettining xasiyetlik san ikenlikini bilgili bolidu. Tonyuquq menggü téshida “...ormanliq we tagh dalilarda qalghanliri yighilip yette yüz boldi... Ilterish qaghan dana, batur bolghanliqi üchün tabghach bilen on yette qétim urush qildi..." (Sartikin, 2005) digendek bayanlar uchraydu. Menggü tash tékistliride yettining 10 we 100 bilen köpeytilgen shekilliri we 17, 27 dégendek shekillirimu alahide gewdilik bolghan.

Biz Turpan wesiqliridimu yettini ulughlashqa da'ir bayanlarni uchritalaymiz. Mesilen "...Chingwugha yette sitir berduq... Yeni babaqtiki saman yapghidin yette parche turquni xalta qilip ewetti... Küzde yette yögem pul qayturimen...” (Turdi, 2002: 41). Tetqiqatchi Abduqadir Turdi yene Ürümchi we Ichki Mongghuldin, Dunxu'angdin, Yekendin tépilghan qedimki Uyghur yéziqidiki wesiqlerdiki yettini ulughlash we chong bilishke da'ir nurghunlighan misallarni bayan qilidu.

Bügünki künde Uyghurlarning turmushida yettini muqeddes bilishke da'ir nurghunlighan adetler bar. Mesilen, kishi ölgendin kéyin uning yette nezirsini qilish, méyitni aldi bilen yette ketmen topa bilen kömüsh we yette kün'giche qéshidin ayrilmasliq. Oghul bala yette yashqa kirgende uning xetne toyini qilish. Yette künde baligha isim quyush. Uyghurlarda yene "su yette domilisa halal" deydighan qarash bar. Uyghurlarda yene "barmaymen dégen tügmen'ge yette qétim bériptu" dégendek maqal-temsiller bar. Xelq éghiz edebiyati eserliride "yette bashliq yalmawuz", "yette batur", "yette melike", "yette qat asman", "yette künlük yol" dégendek ibariler köplep uchraydu. Démek, yettini xasiyetlik bilish aditi Uyghurlarning turmushigha chongqur singip ketken.

Uyghurlar islam dinini qubul qilghandin kéyin, Uyghurlarning turmushida tüptin özgirish boldi. Uyghurlarning islamiyetning ilgiriki étiqad adetliri islam dinining tesiri bilen xéli zor derijide untulghan bolsimu, emma bezi adetler hazirghiche dawamliship kelgen idi. Yette gerche iptida'iy étiqadning mehsuli bolsimu, islam dinidiki yettige munasiwetlik qarashlar bilen zich baghlinip üzlüksiz mewjutluqini saqlap qalghan. Islam dinidimu yettige munasiwetlik bezi qarashlar bar. Mesilen, hej dawamida kebini yette qétim aylinish, namaz oqughanda yette ezani yerge tegküzüsh, dozaxni yette dep teriplesh, qurban héyt namizigha barghuche yolda yette qétim tekbir éytip méngish, it yalap qoyghan qachini yette qétim yuyush, Qur'ani kerimdiki birinchi sürining yette ayettin terkip tépishi qatarliqlar (Yaqup, 1995: 78). Démek, Uyghurlarning eslidiki xasiyetlik sanlar étiqadchiliqi İslam dini qobul qilinghandin kéyinmu özgermigen we İslam dinidiki shu xil qarashlar bilen bille mewjut bulup turghan.

Yuqiriqilardin körüshke boliduki, yette islam dinidila ulughlinidighan san bolmastin, qedimki Uyghurlarning turmushidimu bu xil köz-qarash mewjut bolghan. Bügünki künde Uyghurlar ötküzüp kéliwatqan yette nezirsi emeliyette İslam dinidin kelgen bolmastin Uyghurlarning yiraq qedimki zaman tepekkur we itiqad bilen munasiwetlik sanlarni ulughlashtin kélip chiqqan nezir aditidur.

Bezi tetqatchilirimiz Uyghurlarning yettini ulughlash qarishini Mani dini we Hindi dini bilen baghlap chüshendüridu. Yene bezi tetqiqatchilar yette étiqadini Türk milletlirining astronomiyelik bilimi bilen bille baghlap chüshinidu. Mesilen, tetqiqatchi, proféssor Abdukérim Raxman ependi Uyghurlardiki yettini ulughlash qarishi heqqide "Uyghur folkloridin umumiy bayan" namliq kitabida sirliq san yettining aldi bilen Zara'astir dinida barliqqa kelgenlikini, andin Mani dini arqiliq Uyghurlargha singip kirgenlikini yazidu. Zara'astir dinida yette dane muqeddes tengri bar bolup, Mani dini Zara'astir dini asasida 3esirde barliqqa kelgen. Hemmeylen'ge éniqki, Zara'astir dini miladiyedin ilgiriki 6-esirlerde ijad qilinghan. Zara'astir dini “otni merkez qilghan shaman we tebi'et ilahchiliqi en'enilirini 
asas qilip shekillen'gen" (Muhemmetimin, 1997: 43) bolup, alimlar zara'astir dinining ijad qilinghan waqtini asasen miladiyedin ilgiriki 6-esir dep qaraydu. Cherchendin chiqqan qedimiy qebriler we axiretlik boyumlardiki yette étqadchiliqigha a'it buyumlarning buningdin ilgiriki 3800-yillar burunqi dewirdin qalghanliqini nezerge alghanda Uyghurlarning yettini ulughlash aditining Zara'astir we Shaman dini bilen munasiwetlik ikenlikini bilgili bolidu.

Yette heqqide tetqiqat élip barghan tetqiqatchilardin İltikin Uyghurlardiki xasiyetlik san yettining kélip chiqishi heqqidiki tetqiqatlardin töwendikilerni xulasilep chiqqan: Birinchisi, yettini ulughlash qarishi Zara'astir dinidin kélip chiqqan deydighan qarash; Ikkinchisi, Uyghurlardiki yette muqeddesliki Hindi dinidin Hindilar arqiliq qobul qilinghan dégen qarash. Üchinchisi, yettini muqeddes bilish Shaman dini bilen munasiwetlik deydighan qarash (Iltikin, 2001: 7). Iltikin Uyghurlarning yettini xasiyetlik bilish qarishini Shaman eqidisi bilen munasiwetlik dep qaraydu. Shundaqla muqeddes san yettining qedimki Oral-Altay milletliri arisida peyda bolghan Shaman dinining eng büyük ilahi quyashning ornigha bolghan chüshenche asasida barliqqa kelgenlikini, miladiyidin burunqi 5000-yillarda Türk milletlirining tunji qétim Gherbiy Asiya (Méssopotamiye)ge köchüp bérip, Somirlarni shekillendürishige egiship, Türk milletliridiki bu xil muqeddeschilik chüshenchisining Somér epsaniliride ipadilinishke bashlighanliqini yazidu. Iltikin yene özining bu qarishini tarixiy matériyallar arqiliq ispatlap chiqidu. Eger yuqiriqi qarash bilen bolghanda yettini ulughlash Türkler arqiliq dunyagha tarqalghan bolidu.

Shaman dini bilen munasiwetlik Ölgin Tengri epsaniside Ölgin Tengri (quyash tengrisi) ning yette oghli bar dep qarilidu. Yette oghul yette yultuzni körsitidighan bolup, Uyghurlarning qedimki tebi'et dini-Shaman dini bilen munasiwetlik idi. Abdukérim Raxman ependi kéyin yazghan "Türk xelqliri folklorida muqeddes sanlar chüshenchisi" namliq maqalisida Uyghurlardiki yettini muqeddes bilish qarishining kökke choqunush bilen munasiwetlik ikenlikining éhtimalliqi heqqide pikirini otturigha qoyup, yettini ulughlash qarishining Uyghurlarning Zara'astir dini we Mani dinilirini qobul qilish bilen téximu kücheygenlikini yazidu (Raxman, 1997:40). Tetqiqatchi Meremnisa Metniyaz: "Uyghurlarda xasiyetlik san 'yette' we hepte uqumi toghrisida" namliq maqalisida yettini ulughlash qarishining "astronomiyilik we iptida'iy kaléndarchiliq asasidiki sawat we shu asasta kélip chiqqan adet tüsi" ikenlikini yazidu (Metniyaz, 2003:63). U yene Uyghurlardiki qedimqi hepte uqumining yette yultuzning nami bilen munasiwetlik ikenlikini, yette yultuzning bayqilishining Uyghurlarning iptida'iy astronomiyelik bilimi bilen munasiwetlik ikenlikini otturigha qoyidu.

"Epsaniwi san we muqeddes sanlar sistémisi" namliq maqalide Érnist Kasiri: "Kéyinki GrikRim munejjimliki dewrige kelgende yettige bolghan choqunush yette plantqa bolghan choqunush bilen birleshken. Deslepte, yette künlük dewr bilen yette künlük hepte bu xil munasiwetni körsitip bergen bolmastin, belki tebi'iy halda, üzlüksiz halda bölün'gen"likini otturigha qoyidu (Kasiri, 2001:59).

Omumen yettining xasyetlik sanlar qatarigha kirish heqqide mundaq ikki xil qarash bar. Biri, yette tebi'et dunyasidiki yette shey'i (yultuz) bilen munasiwetlik deydighan qarash. Bezi tarixchilar Türk xelqlirining yettini ulughlishini yette dane shimaliy qutup yultuzi bilen baghlap chüshendüridu. Ularning qarishiche köchmen charwichiliq bilen shughullinidighan milletler muqim olturaqlashmaydighan bolghachqa, tereplerni békitishte yette dane shimaliy qutup yultuzigha tayanghan. "Iptida'iy tepekkur" namliq meshhur eserning apturi Liwi Bolwér dunyadiki nurghun milletlerning yettini ulughlash qarishining kélip chiqishi heqqide iptida'iy kishilerning tesewwurida töt terepning muqeddes sanilidighanliqini, bu töt terep-tötke, asman, zimin we adem özi turiwatqan orun qoshulup yettige köpeygenlikini, shuning bilen qedimqi insanlarning yettini ulughlash qarishining kélip chiqqanliqini yazidu. Yettini ulughlash heqqidiki ikkinchi közqarash: Liwi Bolwér yene Malayaliqlarning yettini 
ulughlash qarishining ilahlar bilen baghlinishliq ikenlikini, ularning itiqad pa'aliyitide yettige baghlinishliq nurghun périxonluq pa'aliyetlirining bolghanliqini otturigha qoyghan (Bolwér, 1981). "Uyghurlarda Shamanizm" namliq kitabning aptori Raxman Abduréhim: "Uyghurlarning yette sanini ulughlash adetliri Uyghur shamanlarning Périxonluq pa'aliyetliride nahayiti gewdilik ipadilinidu. Mesilen 'chiragh' pa'aliyitide yette jup poshkal teyyarlinidu, 'Pére oyuni'da Shaman tughni yette qétim aylinip chiqidu, undin bashqa Shaman yene yette jup poshkalgha yette chiraghni qadap oqush pa'aliyetlirini élip baridu. Shamanning qarishiche, yette jup poshkal-'yette ilah'qa atap qilinghan nezir; yette chiraghyette ilah (yette sultan) ning simwoli hésablinidu. Undin bashqa 'Pére oyuni'da ishlitidighan yette putaqliq derex shéxi, yette xil rengdiki puruchlarmu we depne pa'aliyitide méyitni yerlikke qoyghanda, méyitning qérindashliri lehet ichige yette ketmen topa tashlaydighan adetmu yette sanining muqeddeslikini idiywi menbe qilghan" (Abduréhim, 2006: 284) dep yazidu. Démek, Raxman Abduréhimgha asaslanghanda Uyghurlarning yettini ulughlash qarishi iptida'iy étiqad -Shaman dini bilen munasiwetlik bolidu. Biz meyli arxé'ologiyelik matériyallarni asas qilmayli, meyli Shaman dinining bügünki qalduq hadisiliri heqqide oylanmayli, yettining Uyghurlarning Shaman étiqadi bilen munasiwetlik ikenlikini jezimleshtürimiz.

Uyghurlardiki yigirme we qiriq nezirlirige munasiwetlik yigirme we qiriq sanlirining ulughlinishi heqqide tarixiy pakitlar bir qeder az uchraydu. Yettini muqeddes bilidighan bezi milletlerning aditide yettining hessilik sanighimu ehmiyet béridighan bezi qarashlar mewjut. Uyghurlardiki yigirme nezirning ötküzülüshi (Xoten rayonida) belkim yettning üch hessisi 21 bilen munasiwetlik bolushi mumkin. Yettini üch hessiligende 21 bolidu. Uyghurlarning nezir aditide meyli yette nezirisi bolsun, meyli qiriq nezirsi bolsun yette we qiriq künlerni toluq toshquzmaydu. Bu nuqtidin qarighanda yigirme nezirisi yettining üch hessisi bilen munasiwetlik bolushi mumkin. Chünki "üch"mu Uyghurlarning xasiyetlik sanlirining biri hésablinidu. Doktur Dilmurat Ömer ependi "Altay til sistimisidiki xelqlerning Shaman dini" namliq kitabida Türk xelqliride yettining hessilik sanighimu ehmiyet béridighanliqini yazidu (Ömer, 1995).

Qiriq nezirisining kélip chiqishigha kelsek, Uyghurlar ezeldin qiriqni ulughlap kelgen. Uyghur xelq chöchekliride "qiriq kéche-kündüz toy boluptu”, "qiriq kéche-kündüzlük yol iken" dégendek bayanlar uchraydu. Uyghur qizliri chachlirini qiriq örüm örüydu. Bowaq tughulup qiriq kündin kiyin böshükke sélish murasimi ötküzilidu (bu adet bezi rayonlarda ötküzülmeydu). Xelqimizning en'eniwiy qarishida ayal kishi yenggip qiriq kün'giche dem alidu we kütinidu. Qiriq kündin kéyin qopup, er terepning öyige baridu. Bayan qilsaq edebiyatimizda qiriqqa munasiwetlik nurghun bayanlar uchraydu.

Qiriqni ulughlash qarishining menbesi heqqide proféssor Abdukérim Raxman ependi we tetqiqatchi Raxman Abduréhim iptida'iy insanlarning neziride eng chong saniyalaydighan sanning on ikenlikini, onning ademning ikki qolidiki barmaqlarni sanashtin kelgenlikini, shuning bilen töt terepni ulughlash qarishi buyiche on sanini tötke köpeytip yaki bir-birlep qoshush arqiliq qiriqni keltürüp chiqarghanliqini yazidu (Rahman, 1989). Bu ikki tetqiqatchimizning qarishi "iptida'iy tepekkur" namliq kitabning aptori Liwi Bolwér'ning qarishi bilen birdek, bolup, Liwi Bolwér yuqiriqidek qarashni otturigha qoyidu.

Qiriq muqeddeslikining xelq ichidiki tarqilishigha kelsek, Uyghurlar étiqad qilghan Zara'astir dinidiki qiriq chilten chüshenchisi we islam dinidiki qiriqqa munasiwetlik bayanlar (Islam üchün jeng qilip ölgen qiriq sahabe...qatarliqlar) bilen qoshulup tereqqi qilghan we béyighan bolushi mumkin. Bügünki künde Uyghurlarda méyitning qiriq künige atap nezir ötküzüsh aditi mewjut. 
Démek, Uyghurlar bügünki künde ötküzüp kéliwatqan üch, yette, yigirme, qiriq we yil nezir adetliri ular ulughlap we chong körüp kelgen muqeddes sanlar chüshenchisi bilen munasiwetlik bolup, İslam dini bilen chong baghlinishliqi yoq.

Uyghurlar adem ölüp üch kündin kiyin rohi öydin ayrilmaydu (üch nezirisi ötküzilidu), yette kün'giche uning rohi hoylida bolidu (yette nezirisi ötküzilidu), qiriq kün'giche uning rohi öy bilen qebristan arisida bolidu (qiriq nezirisi ötküzilidu). Bir yil toshqandin kéyin ölgüchining rohi yette qat asmangha chiqip kétidu (yil nezirisi ötküzilidu) dep qaraydu.

Qiziqarliq yéri shuki, Uyghurlardin bashqa Xuyzulardiki bezi nezir-chiraq adetlirimu bezi sanlargha baghlanghan. Mesilen méyit uzitilip yettinji küni bolghanda, Xuyzular bu künni yettisi dep ataydu. Bu küni méyit uzatquchi qoy, toxu öltürüp, poshkal sélip molla axunumlarni we yüz abroyluq kishilerni öyge kélip quran oqushqa, poshkal yéyishke teklip qilidu.... Yigirme yette, ottuz yette künliri bolghanda nezir pa'aliytining kölimi kichik bolidu, bezide axunumni teklip qilmaydu, peqet poshkal sélip qolum-qoshnilargha we namratlargha tarqitip béridu. Qiriqini küni bolghanda Xuyzular ölgenlerning rohi a'ilidin ayrilidu, dep qaraydu. Choqum emel qilish kérek. Etigende axunumni teklip qilip qebre béshida Qur'an oquydu. Oqup bolghandin kéyin axunum, dost-buraderler, uruq-tughqan, yurtdashlarni öyge poshkal yéyishke teklip qilidu, bezisi sediqe qilidu. Shuningdin bashlap, yüzinchi küni, yili, üchinchi yili, oninchi yili, ottuzinchi yilliri burunqidekla qoy soyup, poshkal sélip, bu adetke emel qilidu. Ottuz yil ötüp ketkendin kéyin, chongraq eslesh pa'aliyiti qilmaydu (Wang Zhéngwéy, 2008: 210).

\section{Xulase}

Uyghurlarning muqeddes sanlar chüshenchisining kélip chiqishi bir qeder murekkep bolup, téximu chongqur tetqiq qilishqa toghra kélidu. Omumen qilip éytqanda, Uyghurlar étiqad qilghan dinlar bezi sanlarning ulughlinishigha sewep bolghan. Bolupmu Shaman we islam dinining Uyghurlarning xasiyetlik san chüshenchisige körsetken tesiri tolimu chongqur. Bügünki künde Uyghurlar turmushida Shaman dinining tesiri asasen yuqalghan bolsimu, Shaman dinigha baghlinishliq bezi adetler hélihem mewjut bolmaqta. Bu seweptin, Uyghurlarning xasiyetlik san chüshenchisining yilitiz yenila Shaman dinida. Islam dini bezi sanlarning tekrar qétim sanigha ehmiyet bersimu, biraq sanlarni ulughlashni we choqunushni yaman köridu. Shunglashqa, Uyghurlar turmushdiki muqeddes sanlargha chétishliq murasim we örp-adetler heqqide izden'gen waqtimizda Shaman dinigha munasiwetlik menbelerni estayidil tetqiq qilishqa toghra kélidu. Üch, yette, yigirme, qiriq sanlirigha munasiwetlik étiqadiy adetler bügün'giche Uyghurlar turmushida mewjut bolup, üch, yette, yigirme, qiriq we yil nezirlirining kélip chiqishigha biwaste tesir körsetken. Men yuqirida bayan qilmighandin bashqa yene toqquzgha munasiwetlik bezi adetler qedimdin buyan Uyghurlarning turmushida xéli chongqur derijide mewjut idi. Qisqisi, Uyghurlarning xasiyetlik sanlar chüshenchisi we bu chüshenchilerge baghlanghan örp-adetler Uyghurlar étiqad qilghan Zara'astir dini, Shaman dini, Mani dini we Islam dini qatarliq bir qatar dinlarning, bolupmu Shaman dinining tesirige köp uchrighan we Uyghurlar turmushida ölümge munasiwetlik örp-adetlerning shekillinishige sewep bolghan dep qarashqa bolidu. 


\section{Izahatlar}

ABDURÉHIM, Raxman. (2006). Uyghurlarda Shamanizim (Uygurca). Béyjing: Milletler Neshriyati.

BOLWÉR, Liwi. (1981). Iptida'iy Tepekkur (Çince). Béyjing: Soda Neshriyati.

ILTIKIN. (2001). “Muqeddes San Yette (Uygurca)”. Miras Jurnili, S. 5, bet. 7.

KASIRI, Érnisit. (2001). "Epsaniwi San We Muqeddes Sanlar Sistémisi (Uygurca)". (Terjimiçi: Abduqadir Jalalidin), Miras Jurnili, S. 6, bet. 57-59.

Liu Xuetang. (2009). Shinjangning Tarixtin Ilgiriki Din Tetqiqati (Çince). Béyjing: Milletler Neshriyati.

METNIYAZ, Meremnisa. (2003). "Uyghurlarda Xasiyetlik San Yette We Hepte Uqumi Toghrisida (Uygurca)". Shinjang Ijtima'iy Penler Tetqiqati, S. 1, bet 63.

MUHEMMETIMIN, Abdushükür. (1997). Uyghur Pelsepe Tarixi (Uygurca). Ürümchi: Shinjang Xelq Neshriyati.

ÖMER, Dilmurat. (1995). Altay Til Sistimisidiki Xelqlerning Shaman Dini. Ürümchi: Shinjang Xelq Neshriyati.

RAHMAN, Abdukérim. (1989). Uyghur Folklori Heqqide Bayan (Uygurca). Ürümchi: Shinjang Dashö Neshriyati.

RAXMAN, Abdukérim. (1997). "Türk Xelqliri Folklorida Muqeddes Sanlar Chüshenchisi”. Shinjang Instituti Ilmiy Jurnili (Uygurca), S. 2, bet. 40.

SARTIKIN, Eziz Atawulla. (2005). Yadikarliqlirimizdin Medeniytimizge Nezer (Uygurca). Ürümchi: Shinjang Xelq Neshriyati.

TURDI, Abduqadir. (2002). "Shinjangdin Tépilghan Qedimki Uyghur Yéziqi Wesiqliridiki San Muqeddesliki (Uygurca)". Qeshqer Pidagogika Instituti Jurnili, S. 3, bet. 41-42.

Wang Zhéngwéy. (2008). "Xuyzu Folklori (Çince), Ningshiya Xelq Neshriyati, 2-Ay Neshri. YAQUP, Muxtar. (1995). "Islam Dini We Ka'inattiki Muqeddes San Yette (Uygurca)". Qeshqer Pidagogika Instituti Ilmiy Jurnili, S. 2, bet. 78.

YAQUP, Muxtar. (1999). "Alahide San Üch Toghrisida”. Qeshqer Pidagogika Instituti Ilmiy Jurnili (Uygurca), S. 3, bet. 80-81. 